\title{
Keeping an Open Mind: Cognitive Bias in the Evaluation of an Infant with Posterior-Lateral Rib Fractures
}

\author{
Katie Johnson, ${ }^{1}$ Donald Chris Derauf, ${ }^{1}$ Raymond Stetson, ${ }^{1}$ Paul Galardy, ${ }^{2}$ and \\ Jason Homme ${ }^{1}$ \\ ${ }^{1}$ Department of Pediatric and Adolescent Medicine, Mayo Clinic, Rochester, MN, USA \\ ${ }^{2}$ Department of Pediatric Hematology/Oncology, Mayo Clinic, Rochester, MN, USA \\ Correspondence should be addressed to Jason Homme; homme.jason@mayo.edu
}

Received 19 July 2017; Revised 22 September 2017; Accepted 2 October 2017; Published 26 October 2017

Academic Editor: Georg Singer

Copyright (C) 2017 Katie Johnson et al. This is an open access article distributed under the Creative Commons Attribution License, which permits unrestricted use, distribution, and reproduction in any medium, provided the original work is properly cited.

A four-month-old former premature male is incidentally found to have posterior-lateral rib fractures during evaluation of a febrile illness. This finding led to the initiation of a workup for nonaccidental trauma. A thorough history and physical exam ultimately led to the diagnosis, which was not related to abuse. This case highlights a rare sequela of patent ductus arteriosus repair, cautions medical teams to remain aware of how cognitive bias can affect diagnostic decision-making, and emphasizes the importance of a thorough history, physical exam, and medical record review in cases of suspected nonaccidental trauma.

\section{Introduction}

Posterior rib fractures in infants are classically associated with nonaccidental trauma (NAT) [1-5]. This is attributable to both their location and the developmental stage of infants. Posterior rib fractures typically result from a circumferential squeezing of the infant, which creates a levered force at the articulation of the posterior rib and the vertebral body [6]. It would be unusual to sustain a posterior rib fracture from an accidental mechanism, especially for a child who is nonambulatory. Thus, when pediatricians hear the term "posterior rib fracture," they appropriately place NAT near the top of their differential diagnosis.

This cognitive leap is an example of a heuristic-a diagnostic shortcut that uses clinical acumen to form an ingrained association between a presenting sign or symptom and a likely diagnosis $[5,7]$. Other examples of heuristics in pediatrics include the associations between barky cough and croup, slapped-cheek rash and parvovirus, and thumbprint sign and epiglottitis.

While heuristics add efficiency to the diagnostic process, they are not infallible. Heuristics must be taken into consideration with each individual patient context. For example, other causes of rib fractures in infancy include high-impact trauma $[8,9]$, cardiopulmonary resuscitation $[10,11]$, thoracic surgery [12], birth trauma [13], and even chest physiotherapy [14]. Underlying conditions such as osteogenesis imperfecta, osteopenia of prematurity [15], and rickets [16] must also be considered since they place certain infants at higher risk of fractures than others [9].

The case to follow is that of a former premature male infant incidentally found to have posterior-lateral rib fractures on chest X-ray (CXR) during evaluation of a febrile illness and how a thorough history and physical exam ultimately led to a diagnosis that was not related to abuse.

\section{Case Presentation}

The patient was a four-month-old former 28-and-5/7-week gestation premature twin male admitted directly from an outside facility for further evaluation of fever, increased sleepiness, and new oxygen requirement.

The patient had a history of prolonged course in the neonatal intensive care unit (NICU) and had been discharged approximately two months prior. Upon presentation to an outside emergency department (ED), he had a two-day history of fever and sleepiness and was noted to have oxygen desaturations that prompted the administration of 
supplemental oxygen. Laboratory studies were notable for elevated CRP at $116.4 \mathrm{mg} / \mathrm{L}$ (normal $\leq 4.9 \mathrm{mg} / \mathrm{L}$ ), mild thrombocytosis at $494 \times 10^{3} / \mathrm{mcl}$ (normal $150-350 \times 10^{3} / \mathrm{mcl}$ ), and normal leukocyte count at $12.9 \times 10^{3} / \mathrm{mcl}$ (normal $\left.5.0-15.0 \times 10^{3} / \mathrm{mcl}\right)$.

A CXR was obtained and reviewed by the local treating physician, a third-party teleradiology company contracted by the referring hospital during nonbusiness hours, and the senior pediatric resident at the receiving institution. Consistent between the reports were the absence of pulmonary consolidation (i.e., pneumonia) and the presence of radiopaque patent ductus arteriosus (PDA) clips over the mediastinum. The patient was transferred by ambulance to the receiving hospital.

On admission, the patient was managed with empiric antibiotics, pending the results of blood and urine cultures. Both cultures returned negative for growth. Tests for respiratory syncytial virus and influenza were negative. Pain was managed with scheduled Tylenol, and oxygen support was gradually weaned over the next eight hours.

The morning following admission, the referring physician contacted the primary team to inform them that a staff radiologist had reviewed the CXR and amended the initial report, indicating "there is callus associated with the posterior-lateral left fourth and fifth ribs, sequela of healing/healed fractures. There is no finding suggestive of acute osseous abnormality or pneumothorax" (Figure 1). These findings were confirmed by the staff radiologist at the receiving institution. Review of the patient's medical record indicated that the rib fractures were a new finding compared to the most recent CXR, which had been obtained three months priorly, directly after PDA repair in the NICU.

Upon receipt of the outside radiology report, the primary team became concerned for NAT. A skeletal survey was ordered to evaluate for other fractures, and further history and physical exam were obtained. Physical exam revealed two small, well-healed scars on the infant's left upper back, overlying the site of the fractures. There were no other signs of injury such as cutaneous bruising, scratches, burns, oral trauma, or altered mental status.

When the scars were brought to the attention of the patient's father, he suggested that they may be attributable to the patient's PDA repair. This prompted review of the operative report, which read, "a limited left lateral thoracotomy was performed and the hemithorax entered through the third interspace." The posterior-lateral rib fractures were ultimately attributed to this surgical intervention from PDA repair. The skeletal survey was negative for any other fractures.

\section{Discussion}

This case highlights a rare cause of posterior-lateral rib fractures in infants: surgical PDA repair. There is only one reported case in the literature of rib fractures following percutaneous PDA repair [12], and none reported following surgical PDA repair [17].

PDA repair is a commonly performed procedure, particularly among premature infants. When PDA fails to close naturally or with pharmacologic management, several options

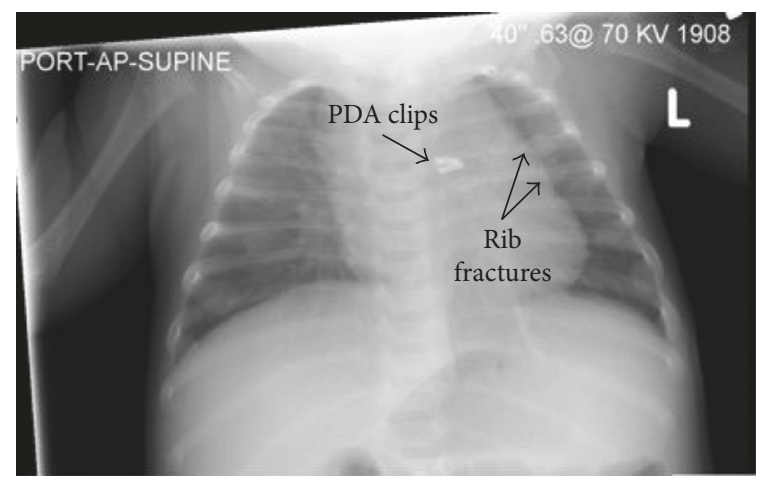

Figure 1: Admission chest X-ray.

are available: surgical repair with posterior-lateral thoracotomy and clip placement or ligation [18]; percutaneous repair (i.e., placement of an occluding device over a wire-guided catheter) $[19,20]$; and less commonly, video-assisted closure [18].

In surgical PDA repair, the two ribs above and the two ribs below the entry interspace are retracted in stages using a rib spreader, to limit rib fractures [18]. In the patient presented here, the surgical team entered through the left third interspace, which was directly above the level of the two identified fractures (i.e., left fourth and fifth ribs). It was concluded that the tension produced by retraction of the fourth and fifth ribs was the cause of the rib fractures, possibly compounded by a component of osteopenia of prematurity or metabolic bone disease. Further discussion with the surgical team suggested that the radiographic findings may not be fractures at all, but instead, periosteal reaction from manipulation of the ribs during the procedure.

This is not only the first report of the association between surgical PDA repair and posterior-lateral rib fractures, but also the first report to our knowledge on the diagnostic overlap between history of PDA repair and initiation of a NAT workup. Indeed, one could argue that a more comprehensive NAT workup was warranted in this case, including a social work assessment, head CT, ophthalmologic exam, labs for abdominal injury, and a follow-up skeletal survey in two weeks. In this case, the patient's family had undergone prior social work assessment while in the NICU, and the primary team felt confident that the posterior-lateral rib fractures with overlying surgical scars on exam and corroborative operative report were sufficient to attribute the fractures to surgical etiology. That being said, the importance of a full NAT evaluation when abuse is suspected cannot be overstated.

An important element of this case is the cognitive bias that can accompany the use of heuristics in medicine. Specifically, we demonstrate how the association between posterior rib fractures in an infant and suspicion of NAT can lead to inaccurate diagnosis if not paired with a careful history, physical exam, and medical record review.

While heuristics serve an important purpose in medicine, they can also predispose clinicians to cognitive bias, which may disrupt accurate clinical reasoning. One such bias is anchoring, in which a clinician commits to a diagnosis early in the workup of a patient at the expense of considering 
other possibilities [7]. In this particular case of a former premature infant with posterior-lateral rib fractures, an open differential diagnosis is of utmost importance, given the complexity of the patient's past medical history, the increased susceptibility of preterm infants to osteopenia and fractures [21-23], and the repercussions of a presumptive diagnosis of NAT. Obtaining further history and a close physical exam helped mitigate the cognitive bias of anchoring to a presumed diagnosis of inflicted trauma.

We present this case as a caution to providers to utilize heuristics carefully, with an understanding of how cognitive bias is inextricably part of the diagnostic process. We hope to impress upon readers the value of a thorough history, physical exam, and medical record review in cases of suspected NAT. Most novel about this case, however, is the association between history of surgical PDA repair and posterior-lateral rib fractures, which had previously not been documented in the literature. This is a rare complication of PDA repair by any method (i.e., surgical or percutaneous), but does occur and should be considered as part of the differential diagnosis for infants with posterior-lateral rib fractures.

\section{Conflicts of Interest}

The authors declare that there are no conflicts of interest regarding the publication of this article.

\section{Acknowledgments}

The authors would like to acknowledge and thank the Mayo Clinic Department of Pediatric and Adolescent Medicine for funding the publication of this manuscript.

\section{References}

[1] A. M. Kemp, F. Dunstan, S. Harrison et al., "Patterns of skeletal fractures in child abuse: systematic review," British Medical Journal, vol. 337, no. 1, p. a1518, 2008.

[2] M. Paddock, A. Sprigg, and A. C. Offiah, "Imaging and reporting considerations for suspected physical abuse (nonaccidental injury) in infants and young children. Part 2: axial skeleton and differential diagnoses," Clinical Radiology, vol. 72, no. 3, pp. 189-201, 2017.

[3] K. A. Barsness, E. S. Cha, D. D. Bensard et al., "The positive predictive value of rib fractures as an indicator of nonaccidental trauma in children," Journal of Trauma-Injury Infection \& Critical Care, vol. 54, no. 6, pp. 1107-1110, 2003.

[4] C. W. Paine, O. Fakeye, C. W. Christian, and J. N. Wood, "Prevalence of abuse among young children with rib fractures: a systematic review," Pediatric Emergency Care, p. 1, 2016.

[5] M. C. Pierce, K. Kaczor, D. Lohr, K. Richter, and S. P. Starling, "A practical guide to differentiating abusive from accidental fractures: an injury plausibility approach," Clinical Pediatric Emergency Mmedicine, vol. 13, no. 3, pp. 166-177, 2012.

[6] P. K. Kleinman and A. E. Schlesinger, "Mechanical factors associated with posterior rib fractures: laboratory and case studies," Pediatric Radiology, vol. 27, no. 1, pp. 87-91, 1997.

[7] D. Kahneman, Thinking, Fast and Slow, Macmillan Publishers, New York, NY, USA, 2011.

[8] S. D. Bixby, A. Abo, and P. K. Kleinman, "High-impact trauma causing multiple posteromedial rib fractures in a child," Pediatric Emergency Care, vol. 27, no. 3, pp. 218219, 2011.

[9] B. Bulloch, C. J. Schubert, P. D. Brophy, N. Johnson, M. H. Reed, and R. A. Shapiro, "Cause and clinical characteristics of rib fractures in infants," Pediatrics, vol. 105, no. 4, p. E48, 2000.

[10] D. Dolinak, "Rib fractures in infants due to cardiopulmonary resuscitation efforts," American Journal of Forensic Medicine \& Pathology, vol. 28, no. 2, pp. 107-110, 2007.

[11] S. Maguire, M. Mann, N. John et al., "Does cardiopulmonary resuscitation cause rib fractures in children? A systematic review," Child Abuse \& Neglect, vol. 30, no. 7, pp. 739-751, 2006.

[12] A. A. Abu Hazeem, M. J. Gillespie, H. Thun et al., "Percutaneous closure of patent ductus arteriosus in small infants with significant lung disease may offer faster recovery of respiratory function when compared to surgical ligation," Catheterization and Cardiovascular Interventions, vol. 82, no. 4, pp. 526-533, 2013.

[13] R. R. van Rijn, R. A. C. Bilo, and S. G. F. Robben, "Birthrelated mid-posterior rib fractures in neonates: a report of three cases (and a possible fourth case) and a review of the literature," Pediatric Radiology, vol. 39, no. 1, pp. 30-34, 2009.

[14] M. Chalumeau, L. Foix-L'Helias, P. Scheinmann et al., "Rib fractures after chest physiotherapy for bronchiolitis or pneumonia in infants," Pediatric Radiology, vol. 32, no. 9, pp. 644-647, 2002.

[15] A. Lucas-Herald, S. Butler, H. Mactier, H. McDevitt, D. Young, and S. F. Ahmed, "Prevalence and characteristics of rib fractures in ex-preterm infants," Pediatrics, vol. 130, no. 6, pp. 1116-1119, 2012.

[16] T. Chapman, N. Sugar, S. Done, J. Marasigan, N. Wambold, and K. Feldman, "Fractures in infants and toddlers with rickets," Pediatric Radiology, vol. 40, no. 7, pp. 1184-1189, 2010.

[17] K. Hutchings, A. Vasquez, D. Price, B. H. Cameron, S. Awan, and G. G. Miller, "Outcomes following neonatal patent ductus arteriosus ligation done by pediatric surgeons: a retrospective cohort analysis," Journal of Pediatric Surgery, vol. 48, no. 5, pp. 915-918, 2013.

[18] P. Valentík, I. C. Omeje, R. Poruban, M. Šagát, and M. Nosál, "Surgical closure of patent ductus arteriosus in pre-term babies," Images in Paediatric Cardiology, vol. 9, no. 2, pp. 27-36, 2007.

[19] C. H. Backes, S. L. Cheatham, G. M. Deyo et al., "Percutaneous patent ductus arteriosus (PDA) closure in very preterm infants: feasibility and complications," Journal of the American Heart Association, vol. 5, no. 2, p. e002923, 2016.

[20] V. V. Dimas, C. Takao, F. F. Ing et al., "Outcomes of transcatheter occlusion of patent ductus arteriosus in infants weighing $\leq 6 \mathrm{~kg}$," JACC: Cardiovascular Interventions, vol. 3 , no. 12, pp. 1295-1299, 2010.

[21] C. Dokos, C. Tsakalidis, A. Tragiannidis, and D. Rallis, "Inside the "fragile" infant: pathophysiology, molecular background, risk factors and investigation of neonatal osteopenia," Clinical Cases in Mineral and Bone Metabolism, vol. 10, no. 2, pp. 86-90, 2013.

[22] S. Nallagonda, M. Nallagonda, and A. Deorukhkar, "Metabolic bone disease of prematurity-an overview," Paediatrics and Child Health, vol. 27, no. 1, pp. 14-17, 2017.

[23] S. E. Rustico, A. C. Calabria, and S. J. Garber, "Metabolic bone disease of prematurity," Journal of Clinical and Translational Endocrinology, vol. 1, no. 3, pp. 85-91, 2014. 


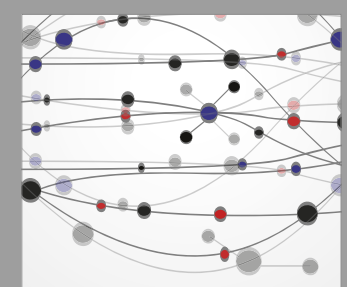

The Scientific World Journal
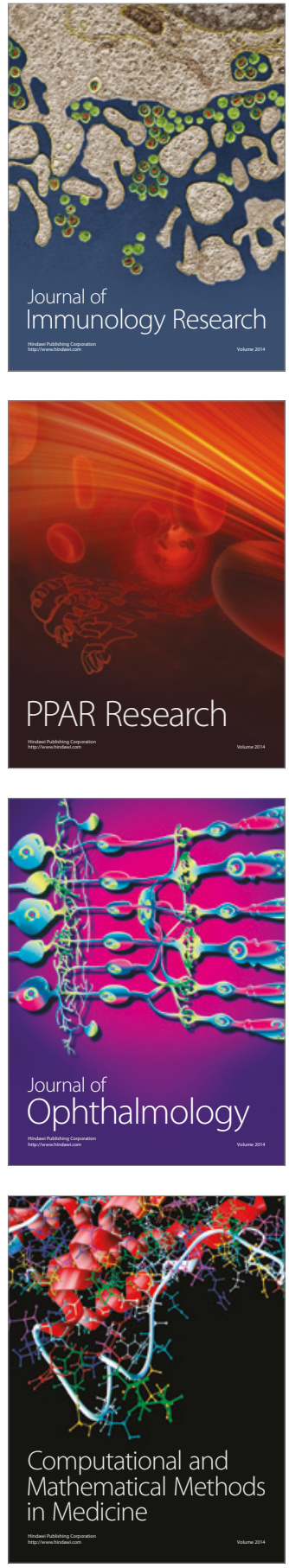

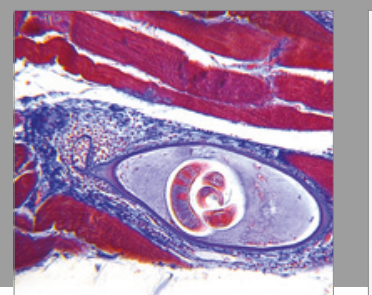

Gastroenterology Research and Practice
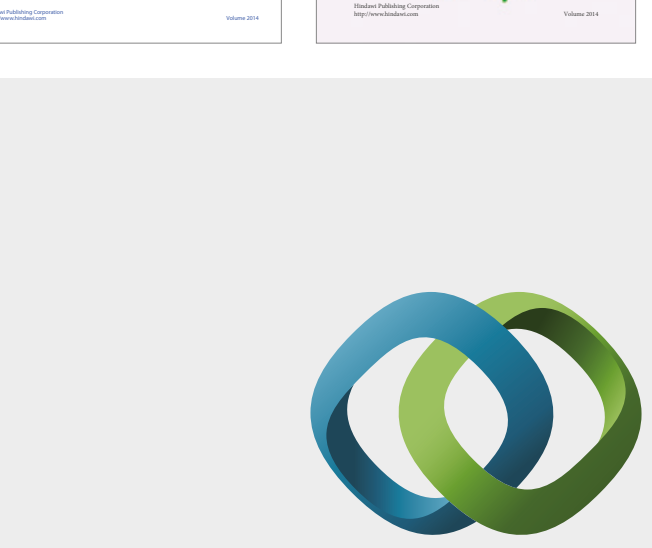

\section{Hindawi}

Submit your manuscripts at

https://www.hindawi.com
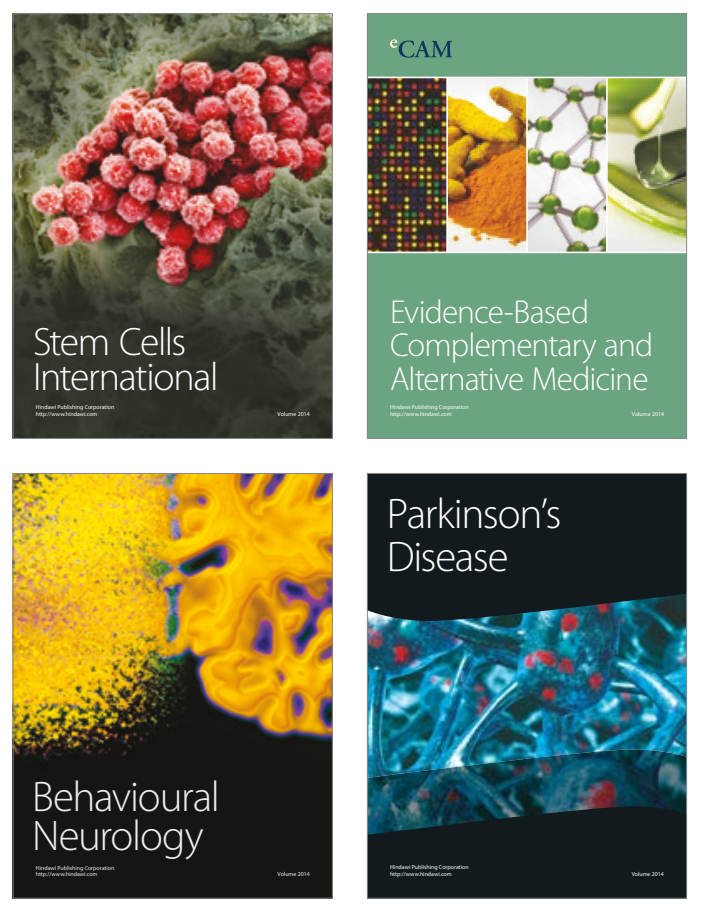
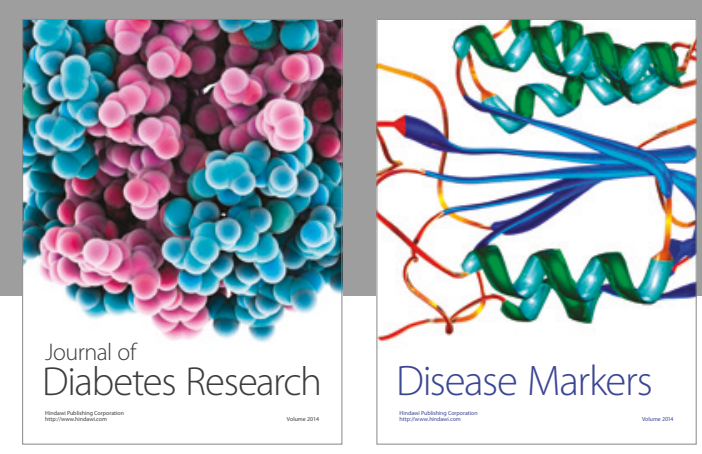

Disease Markers
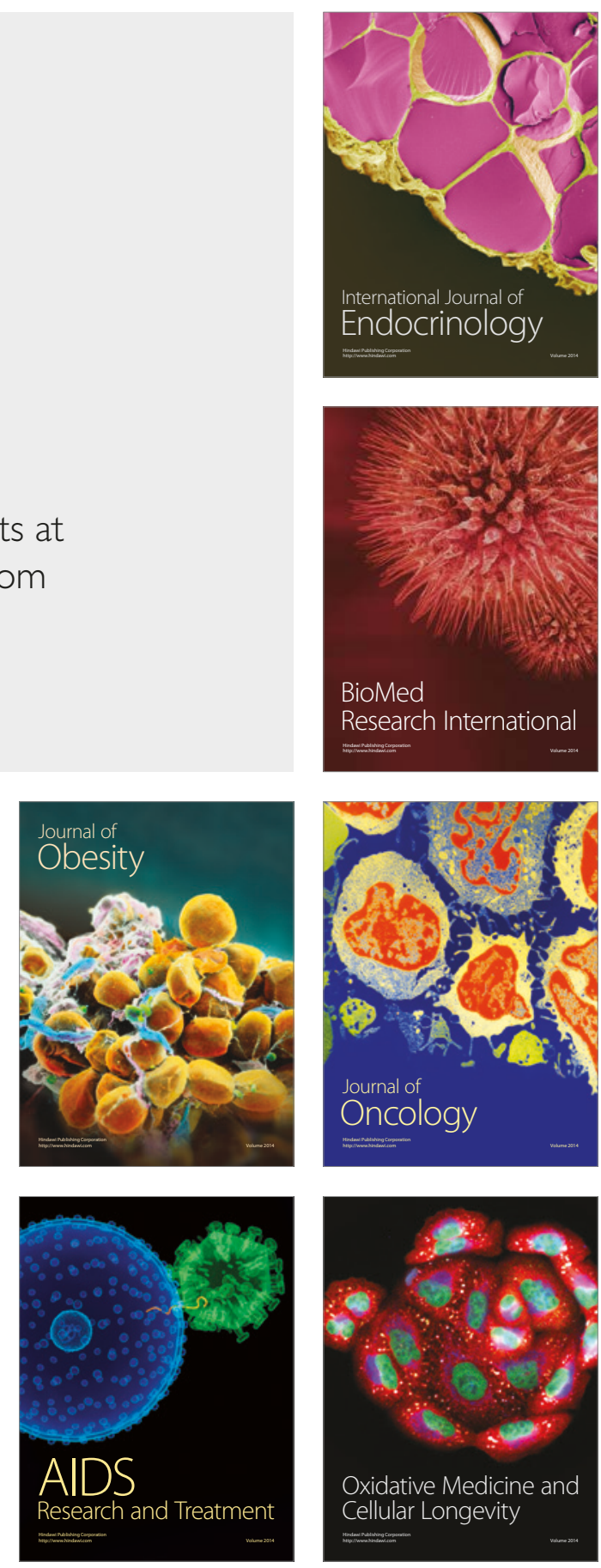